\title{
Critical emergency medicine and the resuscitative care unit
}

\author{
Maria Mermiri ${ }^{1}$, Georgios Mavrovounis ${ }^{1}$, Dimitrios Chatzis ${ }^{2}$, loannis Mpoutsikos ${ }^{2}$, Aristea Tsaroucha $^{2}$, \\ Maria Dova ${ }^{2}$, Zacharoula Angelopoulou ${ }^{3}$, Dimitrios Ragias ${ }^{3}$, Athanasios Chalkias ${ }^{3}$, loannis Pantazopoulos ${ }^{1}$ \\ ${ }^{1}$ Department of Emergency Medicine, Faculty of Medicine, School of Health Sciences, University of Thessaly, Larissa; ${ }^{2}$ Medical School, European University of \\ Cyprus, Nicosia, Cyprus, ${ }^{3}$ Department of Anesthesiology, Faculty of Medicine, School of Health Sciences, University of Thessaly, Larissa, Greece
}

Critical emergency medicine is the medical field concerned with management of critically ill patients in the emergency department (ED). Increased ED stay due to intensive care unit (ICU) overcrowding has a negative impact on patient care and outcome. It has been proposed that implementation of critical care services in the ED can negate this effect. Two main Critical Emergency Medicine models have been proposed, the "resource intensivist" and "ED-ICU" models. The resource intensivist model is based on constant presence of an intensivist in the traditional ED setting, while the ED-ICU model encompasses the notion of a separate EDbased unit, with monitoring and therapeutic capabilities similar to those of an ICU. Critical emergency medicine has the potential to improve patient care and outcome; however, establishment of evidence-based protocols and a multidisciplinary approach in patient management are of major importance.

Key Words: critical care; emergency department; emergency service; intensive care unit; postgraduate training

\section{INTRODUCTION}

Emergency medicine (EM) is the medical specialty that focuses on recognition, evaluation, and coordination of care and treatment of acutely ill patients [1]. Actualization of a structured emergency department (ED) was achieved by U.S. physicians in the 1960s following the realization that management of acutely ill patients required a dedicated group staffed with specialized physicians [2-4]. Critical care medicine (CCM) is a medical specialty developed to provide advanced monitoring and individualized care to patients facing life-threatening conditions. The idea of a specified unit where patients are monitored by specially trained staff was conceptualized during the poliomyelitis epidemic in the early 1950s $[5,6]$. Since then, the intensive care unit (ICU) has become integral to treatment of critically ill patients [7].

Admission of patients to the ICU is often complicated by low bed availability and limited resources [8,9], resulting in substantial delays in ICU admission and increased mortality [1012]. Therefore, implementation of critical care services in the ED could potentially negate these hindrances and improve patient care and outcome.

\section{Review Article}

Received: July 12, 2020

Revised: November 21, 2020

Accepted: November 23, 2020

Corresponding author Maria Mermiri Department of Emergency Medicine, Faculty of Medicine, School of Health Sciences, University of Thessaly, General University Hospital of Larissa, Mezourlo 41110, Larissa, Greece

Tel: +30-6993484469

E-mail:mmermiri@gmail.com

Copyright (๑) 2021 The Korean Society of Critical Care Medicine

This is an Open Access article distributed under the terms of Creative Attributions Non-Commercial License (https:// creativecommons.org/li-censes/by-nc/4.0/) which permits unrestricted noncommercial use, distribution, and reproduction in any medium, provided the original work is properly cited. 


\section{METHODS}

To identify articles for the current literature review, we performed an electronic database search. We searched for published and in print articles in bibliographic databases, including Institute for Scientific Information Web of Science, PubMed, Science Direct, Scopus, Wiley Online Library, Google Scholar, and other international databases and websites. In addition, the literature research involved a manual search of bibliographies of the identified papers and relevant information pertinent to this study. The following keywords were used with the Boolean operators "AND" and "OR" as appropriate: “Critical Care," "Emergency Medicine," “Emergency Department," “Intensive Care Unit," “Models," and “Training." The literature was limited to journal articles written in English. Two reviewers screened all potential references for inclusion. The last literature search was conducted on May 22, 2020.

\section{ICU BED AVAILABILITY}

The aging population, increased patient expectations, and complex medical procedures have led to a greater demand for medical services during the last decade [8]. As a result, the medical community faces essential challenges that hinder patient care, with dire need for bed availability and resources $[9,11]$. Specifically, the demand for critical care services in the U.S. exceeds bed supply [12], and this imbalance is more pronounced in countries with lower bed number to population ratio. European countries like France (11.6 beds per 100,000 population) and Asian countries such as India and China (3 beds per 100,000 population) have substantially fewer available critical care beds than the U.S. (34.6 beds per 100,000 population) [13-15].

The inability to treat critically ill patients due to inadequate personnel and resources is associated with increased mortality and rate of hospital readmission $[10,16]$. The combination of insufficient resources and high demand for medical care has led to implementation of specific criteria and scoring systems to facilitate patient triage $[8,17]$. Some of the factors contributing to the decision for ICU admission are bed availability, severity and reversibility of patient condition, and potential comorbidities $[8,16]$. However, lack of resources increases the complexity of ICU triage decision-making, resulting in fewer ICU admissions [18].

Critically ill patients in the ED that require advanced monitoring and specialized treatment or interventions are significantly affected by ICU overcrowding. Several studies have re-
KEY MESSAGES

- Implementation of Critical Emergency Medicine models has the potential to improve the outcome of critically ill patients presenting to the emergency department.

- Irrespective of specialty, emergency department physicians handling critically ill patients should be highly trained and skilled in resuscitation and in management of life-threatening conditions.

ported that transfer delay longer than 6 hours from ED to ICU is associated with delayed assessment and care, increased probability of medical misconduct, ICU readmission, unfavorable outcome, and early mortality $[19,20]$. Furthermore, an ED length longer than 5 hours was an independent risk factor for poor outcomes in critically ill patients [21-23]. The impact of hospital overcrowding on patient care has become more pronounced during the coronavirus disease 2019 (COVID-19) pandemic due to increased flow of patients through the ED [24]. Patients with acute and critical conditions might be subject to suboptimal care and admission delays as a result of overcrowded departments and general wards [25].

The increased need for ICU beds has led to development of the "ICU without walls" model [26]. In this model, management of critically ill patients in the general ward is optimized by collaboration between healthcare personnel specialized in CCM and ward physicians within the context of a rapid response system (RRS) or medical emergency team (MET). The latter is a small group of individuals specialized in emergency and critical care, which is alerted for assessment of a critically ill patient [27]. In addition to on-site resuscitation, the MET can recognize patients who need to be admitted to the ICU. Research has shown that implementation of RRSs/METs in hospitals is associated with decreased mortality and reduced rates of cardiopulmonary arrest episodes outside of the ICU [28]. The "ICU without walls" model might improve the outcomes of acutely ill ward patients, and increasing evidence suggests that a similar model can be used efficiently for the ED [26]. However, more recent models have been developed during the last decade.

\section{EMERGENCY DEPARTMENT INTENSIVIST}

The Society for Academic Emergency Medicine was the first to propose designated "emergency centers" where critical care services are provided to acutely ill patients [29]. Patients are transported to the emergency center for initial diagnosis and 
management before potential ICU admission. These centers fulfill specific critical treatment criteria including appropriate ICU equipment and specialized healthcare personnel. In this context, a "resource intensivist" model was proposed in 2012 and is based on the constant presence of a physician trained in intensive care in the $\mathrm{ED}$ [30]. ED intensivists are responsible for management of critically ill patients until their admission to the ICU and advise their colleagues on controversial matters concerning treatment of severe diseases. Notably, ED intensivists can continue management of patients after ICU admission or provide their services in other areas of the hospital, including the emergency department-integrated intensive care unit (ED-ICU). This unit is located within the ED and possesses monitoring and therapeutic capabilities similar to those of an ICU. Such a unit enables ED physicians and intensivists to deliver critical care services irrespective of ICU bed availability, decreasing the adverse effects of hospital overcrowding [31]. Two ED-ICU models have been described, the "hybrid" and the "stand-alone ED-ICU."

The hybrid model can be implemented in institutions where the ED incorporates dedicated resuscitation bays [30]. Here, inclusion of an ED intensivist enables management of critically ill patients beyond the first hour after resuscitation, constituting a hybrid "resuscitation bay and ED-ICU" (RED-ICU or resuscitative care unit). The ED intensivist leads a group of emergency physicians (EPs) and appropriately trained nursing staff and ensures that the team follows the established protocols for management of critically ill patients arriving in the ED. This model was utilized in a study including critically ill patients who required time-sensitive surgical interventions [32]. The authors reported that implementation of the model facilitated transfer to the operating theater or ICU and improved patient outcomes. A 22-bed RED-ICU is in operation at Stony Brook University Medical Center and incorporates an Acute Critical Care Subunit encompassing eight beds, while the remaining beds are reserved for ED patients in need of further evaluation, monitoring, and treatment [33]. This unit functions as an extension of the ED and is staffed by EPs and resuscitation fellows.

The stand-alone ED-ICU model consists of an isolated unit within the ED containing ICU beds and appropriate equipment [33]. Critically ill patients in the ED are stabilized and

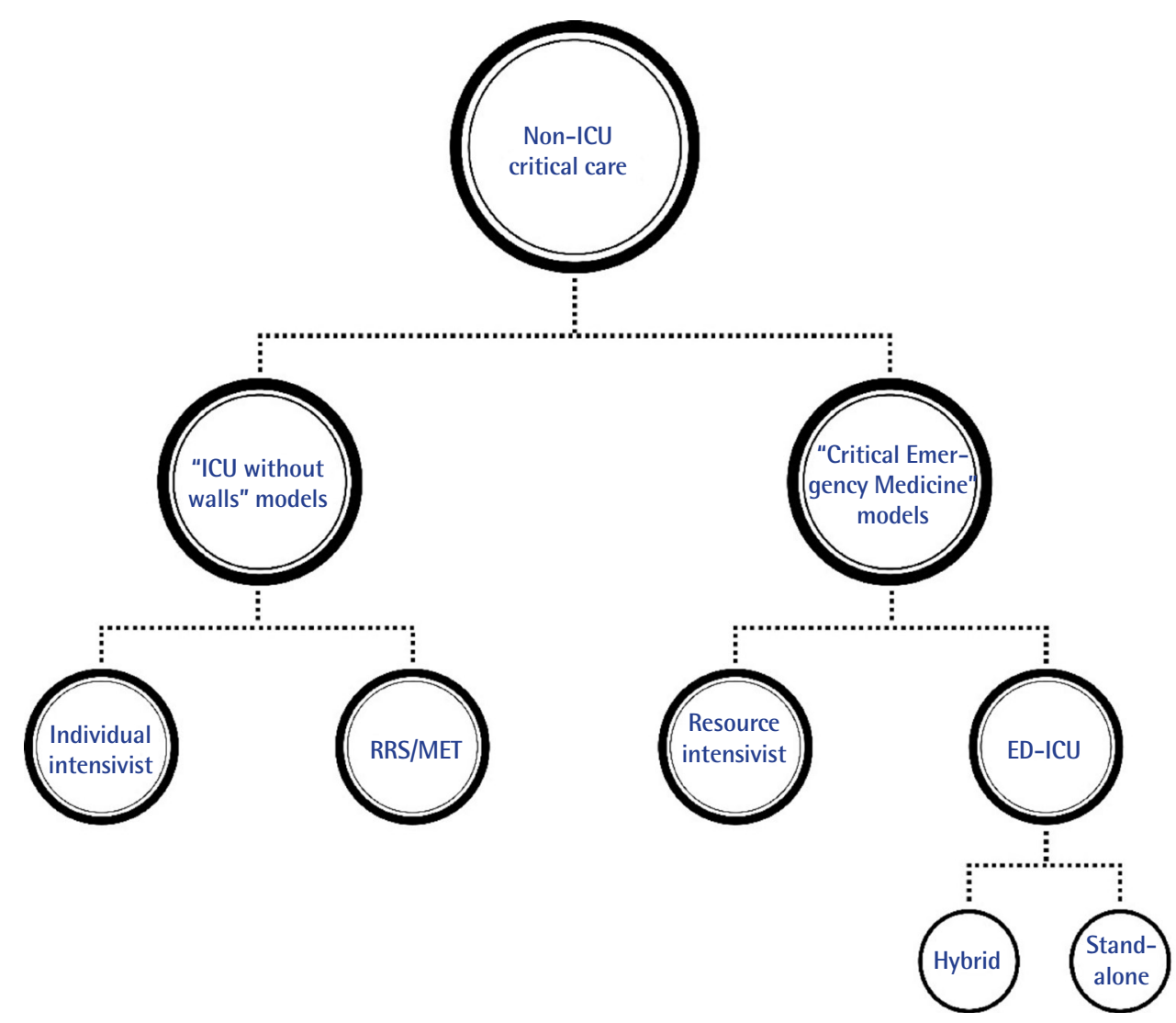

Figure 1. Non-intensive care unit (non-ICU) critical care models. RRS: rapid response system; MET: medical emergency team; ED-ICU: emergency department-integrated intensive care unit. 
admitted to the ED-ICU for further treatment. This model negates the issue of ICU admission delay, although it might not be cost-effective due to the need for de-novo creation of ICU beds. A stand-alone ED-ICU, termed the emergency critical care center (EC3), has been created and established at the University of Michigan University Hospital [20]. It is a ninebed ICU within the ED, containing five resuscitation bays and four designated trauma beds. The EC3 provides ICU-level care by physicians trained in critical care, independent of their specialty. The University of Michigan EC3 was the site of a large observational retrospective cohort study from 2012 to 2017 [20]. In that study, patients were divided into pre-EC3 (20122015) and post-EC3 (2015-2017) groups. Using electronic patient records, 30-day mortality and ICU admission rate were calculated for both groups. The authors concluded that EDICU implementation was associated with lower 30-day mortality and reduced rates of ED to ICU transfer. The University of Pennsylvania Resuscitation and Critical Care Unit is another example of the stand-alone ED-ICU model [33]. This fivebed ICU is located within the ED, and its staff includes exclusively certified intensivists and EPs with advanced resuscitation training.

Observational retrospective cohort studies have shown that ED-ICUs are effective in lowering mortality and in improving duration in the ED [20,32]. However, randomized controlled trials along with cost-effective analyses are needed to conclude whether ED-ICUs have a significant impact on patient care (Figure 1) [30].

\section{CRITICAL EMERGENCY MEDICINE}

Critical emergency medicine is an emerging area focusing on resuscitation of unstable ED patients and refers to acute medical care of patients in medical emergency that pose an immediate threat to life, irrespective of location, such as those with sepsis or shock [34]. The term was introduced in 2010 by the Scandinavian Society of Anaesthesiology and Intensive Care Medicine, describing the roles and competencies of anesthesiologists and intensive care physicians in caring for patients with life-threatening illness or injury. One reason the term was introduced was to distinguish these core activities from the broader internationally-recognized medical specialty of emergency medicine [35]. In 2016, the European Board of Anaesthesiology and European Society of Anaesthesiology formally adopted the term "to define anesthesiologists' competencies and role in the acute management of life-threatening emergencies," which had previously been referred to in the anesthesiology specialty [36]. Physicians providing critical care services in the ED are confronted with life-threatening acute illnesses and should have specialized resuscitation knowledge and skills [37]. As previously mentioned, treatment of critically ill patients in the ED involves a variety of medical specialties or subspecialties, such as intensivists, EPs, and anesthesiologists [30]. However, no agreement has been reached as to which specialty is most qualified to undertake the care of these patients.

In view of this debate, the European Society of Anaesthesiology and the European Board of Anaesthesiology issued a statement declaring that management of critically ill patients in the ED should be the responsibility of the anesthesiologist [36]. Anesthesiologists are trained in critical care, emergency, and pain medicine and acquire the necessary skills for coping with life-threatening situations under the controlled environment of the operating room. They additionally proposed that anesthesiologists are suitable for undertaking leadership roles during emergencies since they are trained to do so in the operating room. However, several authors refuted this concept, suggesting that EPs are essential in assessment and diagnosis of critically ill patients in the ED, especially when overcrowding becomes an issue $[33,37,38]$. EPs are specifically trained to establish the correct diagnosis among a plethora of possible conditions and are experienced in selecting the appropriate diagnostic work-up, while also being trained in resuscitative interventions $[39,40]$. In the era of the COVID-19 pandemic, EPs play an essential role in timely triage of patients suspected to be infected and in need of advanced monitoring and care [41].

It has been proposed that patient care in the ED can be improved by the presence of an ED intensivist, who can provide additional expertise in resuscitation and management of critically ill patients. Intensivists also can facilitate protocol establishment and implementation in the ED setting. Finally, they can train less experienced personnel and perform simulation courses in advanced resuscitation techniques and critical care [30]. Regardless of their primary specialty, the ED resuscitationist must have extensive knowledge and training in resuscitation science, which is the most important prerequisite for provision of high-quality critical emergency medicine.

\section{TRAINING IN CRITICAL EMERGENCY MEDICINE}

An assortment of training pathways has become available to physicians interested in critical emergency medicine [42]. Training can be acquired through residency rotations, courses, or 
postgraduate studies. The majority of Emergency Medicine and Anesthesia Residency Programs provide training in crucial critical care procedures, such as airway management, central venous access, and fluid resuscitation [43]. Critical emergency medicine physicians should be proficient in invasive monitoring techniques, including invasive blood pressure monitoring, central venous pressure measurement, and pulmonary artery catheterization [44]. These skills can be acquired during specialization or through subspecialization [45].

The Scandinavian Society of Anaesthesiology and Intensive Care Medicine introduced a novel 2-year post-specialist training course for anesthesiologists interested in critical emergency medicine [35]. The focus of this program is to provide anesthesiologists with the appropriate skills for undertaking treatment of critically ill or injured patients in the pre-, in-, and inter-hospital settings. It also aims to improve knowledge of the systems required for provision of critical care in those settings and to promote quality assurance programs and academic research. Anesthesiologists who have documented interest and experience in critical care are eligible to apply. Other specialists are considered suitable to apply for this program as long as they have at least 2 years of experience in anesthesiology as well as board-qualified knowledge of resuscitation and life support. Participants are required to undertake four, 4-day residential courses and to organize an exchange period with a clinic or department of a different country. Trainees are obligated to participate in a scientific project spanning at least 2 years and to work simultaneously in an emergency critical care setting.

The post-graduate study program (MSc) "resuscitation" of the National and Kapodistrian University of Athens, Greece, is a training program that provides high-quality expertise in the field of resuscitation [46]. This MSc is not taught in any other European or U.S. academic institution and focuses on training healthcare personnel in the developing field of resuscitation science and introducing appropriate methods for high-quality resuscitation-related research. Specific areas of research interest include successful management of cardiac arrest, prevention of life-threatening complications, and critical patient care. The program aims to provide expertise in resuscitative techniques, to train physicians to cope with possible clinical dilemmas, and to familiarize participants with management of successfully resuscitated patients in the ED or ICU. A summary of the opportunities available for CCM and resuscitation subspecialization is presented in Table 1.

Table 1. Critical emergency medicine and resuscitation training pathways

\begin{tabular}{|c|c|c|c|}
\hline Country & $\begin{array}{c}\text { Option for critical emergency medicine } \\
\text { training for EPS }\end{array}$ & $\begin{array}{l}\text { Option for resuscitation } \\
\text { training }\end{array}$ & Comment \\
\hline USA & $\begin{array}{l}\text { 1. Internal medicine critical care fellowship } \\
\text { 2. Anesthesiology critical care fellowship } \\
\text { 3. Surgical critical care fellowship } \\
\text { 4. Neurocritical care fellowship }\end{array}$ & Resuscitation fellowship & - \\
\hline UK & $\begin{array}{l}\text { 1. Dual training (EM and intensive care) } \\
\text { 2. Critical care fellowship }\end{array}$ & Resuscitation fellowship ${ }^{b}$ & $\begin{array}{l}{ }^{a} 8.5 \text { Years for } \mathrm{EM} \text { and intensive care training } \\
{ }^{\mathrm{b}} \mathrm{EP} \text {, acute care physicians, intensive care } \\
\text { physicians, anesthesiologists }\end{array}$ \\
\hline Canada & Critical care fellowship & $\begin{array}{l}\text { Resuscitation and reanima- } \\
\text { tion fellowship }\end{array}$ & - \\
\hline Saudi Arabia & Critical care fellowship & - & - \\
\hline Australia & Critical care fellowship & - & - \\
\hline South Africa & Critical care fellowship & - & - \\
\hline Greece & Critical care fellowship ${ }^{c}$ & $\begin{array}{l}\text { Post-graduate study program } \\
\text { (MSc) "resuscitation"d }\end{array}$ & $\begin{array}{l}\text { 'Internists, general surgeons, anesthesiologists, } \\
\text { cardiologists, and pulmonologists can sub- } \\
\text { specialize in both critical care and EM. } \\
{ }^{d} \text { Available for physicians of all specialties }\end{array}$ \\
\hline China & Critical care fellowship (pulmonary and critical care) & - & - \\
\hline $\begin{array}{l}\text { Scandinavian } \\
\text { countries }\end{array}$ & - & - & $\begin{array}{l}\text { Norway, Finland, Denmark, Sweden, Iceland } \\
\text { Post-specialist training in critical emergency } \\
\text { medicine, available only for anesthesiologists }\end{array}$ \\
\hline
\end{tabular}

EP: emergency physician; EM: emergency medicine.

${ }^{\text {a-d }}$ Each footnote of Option is specifically explained in the same footnote of Comment. 


\section{WHAT LIES AHEAD?}

Critical emergency medicine has the potential to greatly improve the care and outcome of critically ill patients [34]. Nevertheless, several issues must be addressed to standardize and optimize patient care. A consensus on specialties able to practice critical emergency medicine seems mandatory [44]. Considering that critically ill ED patients benefit greatly from a team approach, inclusion of anesthesiologists, intensivists, and EPs seems ideal, with the criteria of (sub)specialization and extensive training in resuscitation science. In countries in which emergency medicine is not a distinct (sub)specialty, critical emergency medicine can be provided by the acute care anesthesiologist [38]. In such countries, the National Societies of Anesthesiology must embed critical emergency medicine and personalized physiology-guided treatment strategies into their training programs [32].

\section{CONCLUSION}

Critical emergency medicine is an emerging field and is anticipated to significantly improve access to high-quality acute care worldwide. Official training curricula must be established to facilitate (sub)specialization and promote evidence-based resuscitation practices in the critically ill ED patient. The ED resuscitation unit can offer high-quality care in critically ill patients, offering an infrastructure for resuscitation.

\section{CONFLICT OF INTEREST}

No potential conflict of interest relevant to this article was reported.

\section{ORCID}

Maria Mermiri https://orcid.org/0000-0001-9057-7142 Georgios Mavrovounis https://orcid.org/0000-0001-7595-5598 Ioannis Mpoutsikos https://orcid.org/0000-0002-1174-6268 Maria Dova https://orcid.org/0000-0002-1998-4121 Ioannis Pantazopoulos https://orcid.org/0000-0002-8846-519X

\section{AUTHOR CONTRIBUTIONS}

Conceptualization: all authors. Methodology: MM, GM, DC, IM, AT. Project administration: PI. Visualization: MM, MG. Writing-original draft, review, \& editing: all authors.

\section{REFERENCES}

1. Holliman CJ, Mulligan TM, Suter RE, Cameron P, Wallis L, Anderson PD, et al. The efficacy and value of emergency medicine: a supportive literature review. Int J Emerg Med 2011;4:44.

2. Groenland CN, Termorshuizen F, Rietdijk WJ, van den Brule J, Dongelmans DA, de Jonge E, et al. Emergency department to ICU time is associated with hospital mortality: a registry analysis of 14,788 patients from six university hospitals in The Netherlands. Crit Care Med 2019;47:1564-71.

3. American Academy of Emergency Medicine. History [Internet]. Milwaukee (WI): American Academy of Emergency Medicine; 2020 [cited 2020 Apr 25]. Available from: https://www. aaem.org/about-us/our-values/history.

4. Suter RE. Emergency medicine in the United States: a systemic review. World J Emerg Med 2012;3:5-10.

5. Hillman K. Critical care without walls. Curr Opin Crit Care 2002;8:594-9.

6. Reisner-Sénélar L. The birth of intensive care medicine: Björn Ibsen's records. Intensive Care Med 2011;37:1084-6.

7. Smith G, Nielsen M. ABC of intensive care: criteria for admission. BMJ 1999;318:1544-7.

8. Orsini J, Blaak C, Yeh A, Fonseca X, Helm T, Butala A, et al. Triage of patients consulted for ICU admission during times of ICU-bed shortage. J Clin Med Res 2014;6:463-8.

9. Halpern SD. ICU capacity strain and the quality and allocation of critical care. Curr Opin Crit Care 2011;17:648-57.

10. O'Callaghan DJ, Jayia P, Vaughan-Huxley E, Gribbon M, Templeton M, Skipworth JR, et al. An observational study to determine the effect of delayed admission to the intensive care unit on patient outcome. Crit Care 2012;16:R173.

11. Long EF, Mathews KS. The boarding patient: effects of ICU and hospital occupancy surges on patient flow. Prod Oper Manag 2018;27:2122-43.

12. Halpern NA, Pastores SM. Critical care medicine in the United States 2000-2005: an analysis of bed numbers, occupancy rates, payer mix, and costs. Crit Care Med 2010;38:65-71.

13. Rhodes A, Ferdinande P, Flaatten H, Guidet B, Metnitz PG, Moreno RP. The variability of critical care bed numbers in Europe. Intensive Care Med 2012;38:1647-53.

14. Phua J, Faruq MO, Kulkarni AP, Redjeki IS, Detleuxay K, Mendsaikhan N, et al. Critical care bed capacity in Asian countries and regions. Crit Care Med 2020;48:654-62.

15. Wallace DJ, Angus DC, Seymour CW, Barnato AE, Kahn JM. Critical care bed growth in the United States: a comparison of regional and national trends. Am J Respir Crit Care Med 2015; 191:410-6. 
16. Christ M, Grossmann F, Winter D, Bingisser R, Platz E. Modern triage in the emergency department. Dtsch Arztebl Int 2010;107:892-8.

17. Sprung CL, Danis M, Iapichino G, Artigas A, Kesecioglu J, Moreno $\mathrm{R}$, et al. Triage of intensive care patients: identifying agreement and controversy. Intensive Care Med 2013;39:1916-24.

18. Robert R, Coudroy R, Ragot S, Lesieur O, Runge I, Souday V, et al. Influence of ICU-bed availability on ICU admission decisions. Ann Intensive Care 2015;5:55.

19. Chalfin DB, Trzeciak S, Likourezos A, Baumann BM, Dellinger RP; DELAY-ED study group. Impact of delayed transfer of critically ill patients from the emergency department to the intensive care unit. Crit Care Med 2007;35:1477-83.

20. Gunnerson KJ, Bassin BS, Havey RA, Haas NL, Sozener CB, Medlin RP Jr, et al. Association of an emergency departmentbased intensive care unit with survival and inpatient intensive care unit admissions. JAMA Netw Open 2019;2:e197584.

21. Carr BG, Kaye AJ, Wiebe DJ, Gracias VH, Schwab CW, Reilly PM. Emergency department length of stay: a major risk factor for pneumonia in intubated blunt trauma patients. J Trauma 2007;63:9-12.

22. Hellyer TP, Ewan V, Wilson P, Simpson AJ. The Intensive Care Society recommended bundle of interventions for the prevention of ventilator-associated pneumonia. J Intensive Care Soc 2016;17:238-43.

23. Samra SR, Sherif DM, Elokda SA. Impact of VAP bundle adherence among ventilated critically ill patients and its effectiveness in adult ICU. Egypt J Chest Dis Tuberc 2017;66:81-6.

24. Guan WJ, Ni ZY, Hu Y, Liang WH, Ou CQ, He JX, et al. Clinical characteristics of coronavirus disease 2019 in China. N Engl J Med 2020;382:1708-20.

25. Cao Y, Li Q, Chen J, Guo X, Miao C, Yang H, et al. Hospital emergency management plan during the COVID-19 epidemic. Acad Emerg Med 2020;27:309-11.

26. Gordo F, Abella A. Intensive care unit without walls: seeking patient safety by improving the efficiency of the system. Med Intensiva 2014;38:438-43.

27. Devita MA, Bellomo R, Hillman K, Kellum J, Rotondi A, Teres $D$, et al. Findings of the first consensus conference on medical emergency teams. Crit Care Med 2006;34:2463-78.

28. Winters BD, Weaver SJ, Pfoh ER, Yang T, Pham JC, Dy SM. Rapid-response systems as a patient safety strategy: a systematic review. Ann Intern Med 2013;158(5 Pt 2):417-25.

29. Goldfrank L, Henneman PL, Ling LJ, Prescott JE, Rosen C, Sama A. Emergency center categorization standards. Acad Emerg Med 1999;6:638-55.

30. Weingart SD, Sherwin RL, Emlet LL, Tawil I, Mayglothling J,
Rittenberger JC. ED intensivists and ED intensive care units. Am J Emerg Med 2013;31:617-20.

31. Gerber DR. Structural models for intermediate care areas: one size does not fit all. Crit Care Med 1999;27:2321-3.

32. Scalea TM, Rubinson L, Tran Q, Jones KM, Rea JH, Stein DM, et al. Critical care resuscitation unit: an innovative solution to expedite transfer of patients with time-sensitive critical illness. J Am Coll Surg 2016;222:614-21.

33. Leibner E, Spiegel R, Hsu CH, Wright B, Bassin BS, Gunnerson $\mathrm{K}$, et al. Anatomy of resuscitative care unit: expanding the borders of traditional intensive care units. Emerg Med J 2019; 36:364-8.

34. Nee P, Andrews F, Rivers E. Critical care in the emergency department: introduction. Emerg Med J 2006;23:560.

35. Søreide E, Kalman S, Åneman A, Nørregaard O, Pere P, Mellin-Olsen J, et al. Shaping the future of Scandinavian anaesthesiology: a position paper by the SSAI. Acta Anaesthesiol Scand 2010;54:1062-70.

36. De Robertis E, Böttiger BW, Søreide E, Mellin-Olsen J, Theiler $\mathrm{L}$, Ruetzler K, et al. The monopolisation of emergency medicine in Europe: the flipside of the medal. Eur J Anaesthesiol 2017;34:251-3.

37. Huang DT, Osborn TM, Gunnerson KJ, Gunn SR, Trzeciak S, Kimball E, et al. Critical care medicine training and certification for emergency physicians. Crit Care Med 2005;33:2104-9.

38. Wilhelms D, Sjöberg F, Chew M. Emergency medicine is about collaboration, not monopolisation. Eur J Anaesthesiol 2018; 35:231-2.

39. Graham CA. Advanced airway management in the emergency department: what are the training and skills maintenance needs for UK emergency physicians? Emerg Med J 2004;21:14-9.

40. Buscher H. Who takes the lead in critically ill patients? Eur J Anaesthesiol 2018;35:232-3.

41. Freund Y. The challenge of emergency medicine facing the COVID-19 outbreak. Eur J Emerg Med 2020;27:155.

42. Aslaner MA. A reality in emergency medicine: subspecialization. Eurasian J Emerg Med 2017;16:144-7.

43. Hockberger RS, Binder LS, Graber MA, Hoffman GL, Perina DG, Schneider SM, et al. The model of the clinical practice of emergency medicine. Ann Emerg Med 2001;37:745-70.

44. Rivers EP, Nguyen HB, Huang DT, Donnino MW. Critical care and emergency medicine. Curr Opin Crit Care 2002;8:600-6.

45. Johnson NJ, Maher PJ, Badulak J, Luks AM. The transition from emergency medicine resident to critical care fellow: a road map. AEM Educ Train 2017;1:116-23.

46. Anazoogonisiekpa.gr. MSc resuscitation [Internet]. 2020 [cited 2020 Jul 9]. Available from: https://anazoogonisiekpa.gr. 